\title{
Hepatoprotective Effect of Ajwa Date Seed and Simvastatin in Non Alcoholic Fatty Liver Disease (NAFLD) Murine Model of Butter Fed Dyslipidemia
}

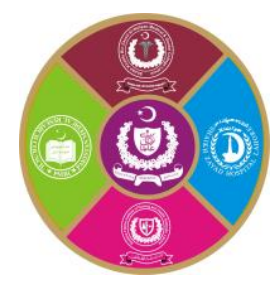

${ }^{1}$ Farooq Sultan, ${ }^{2}$ Saba Anwar, ${ }^{3}$ Saadia Shahzad Alam, ${ }^{4}$ Hassan Farooq, ${ }^{1}$ Samina Kausar, ${ }^{5}$ Tehreem Zulfiqar

${ }^{1}$ Department of Pharmacology \& Therapeutics, Gujranwala Medical College, Gujranwala

${ }^{2}$ Women Medical Officer, Rural Health Centre Hadali, Khushab

${ }^{3}$ Department of Pharmacology \& Therapeutics, Shaikh Zayed Medical Complex, Lahore

${ }^{4}$ Department of Pharmacology \& Therapeutics, Khawaja Muhammad Safdar Medical College, Sialkot

${ }^{5}$ Department of Pharmacology \& Therapeutics, Nishtar Medical University, Multan

\begin{abstract}
Introduction: Non-alcoholic fatty liver disease (NAFLD) is a dangerous complication of hyperlipidemia which may lead to life threatening hepatitis and hepatic cirrhosis. Internationally, Prevalence of non-alcoholic fatty liver disease (NAFLD) is $20 \%$ in general population while $75 \%$ in the obese population of USA. ${ }^{1}$ Side effects of allopathic drugs have led the research towards natural products to find out their potential for treatment of NAFLD. Aims \& Objectives: Aim of this study was evaluation of hepatoprotective effects of Ajwa date seed and Simvastatin against NAFLD. Place and duration of study: Study was conducted at animal house of UHS, Lahore and completed in 6 months. Material \& Methods: 40 rats were procured and segregated into 5 groups with 8 rats in each group. Group 1 was healthy control and group 2 was disease control. Group 3, 4 and 5 were administered Simvastatin $10 \mathrm{mg} / \mathrm{kg} /$ day, aqueous Ajwa date seed extract (ADSE) $8 \mathrm{ml} / \mathrm{kg} / \mathrm{day}$ and combination of Simvastatin $5 \mathrm{mg} / \mathrm{kg} / \mathrm{day}$ and aqueous ADSE $4 \mathrm{ml} / \mathrm{kg} / \mathrm{day}$ respectively. Total study duration was 36 days. First 15 days were for induction of hyperlipidemia while treatment was given for remaining 21 days. Serum total cholesterol (TC), triglycerides (TG) and liver functions tests (Serum ALT, AST and ALP) were performed at day 0, 15 and 36 of study. Data was entered on SPSS 23.0 software and analyzed by one way ANOVA and post hoc tuckey's tests. P-value of less than 0.05 was considered significant. Results: Aqueous Ajwa date seed extract (ADSE), Simvastatin and their combination showed significant TC and TG lowering and hepatoprotective effects against NAFLD. However, individual drugs were more effective than the combination. Conclusion: Ajwa date seed can be used for the treatment of NAFLD.
\end{abstract}

Key words: Ajwa date seed, Hyperlipidemia, Simvastatin

\section{INTRODUCTION}

\section{$\mathrm{H}$} yperlipidemia or dyslipidemia is a metabolic disorder characterized by elevated levels of serum total cholesterol (TC), triglycerides (TG) and low density lipoproteins (LDL) or reduced levels of high density lipoproteins (HDL). ${ }^{2}$ Hyperlipidemia is a leading cause of non-alcoholic fatty liver disease (NAFLD) in which large amount of fats in the form of vacuoles precipitate in the hepatocytes through steatosis. $^{3}$ It may result in the inflammation of hepatocytes and finally, life threatening hepatitis. ${ }^{4,5}$ Prevalence of NAFLD is $20 \%$ in general population while $75 \%$ in the obese population of USA. ${ }^{1}$ Therefore, mandatory requirement of therapeutic intervention arises to combat the consequences of NAFLD. Statins are the most widely prescribed and the most effective agents for the treatment of hyperlipidemia and its associated complications like NAFLD. ${ }^{6}$ They are the inhibitors of 3-hydroxy 3methlylglutaryl coenzyme A (HMG-CoA) reductase enzyme which catalyzes the rate limiting step of cholesterol synthesis. ${ }^{7}$ Simvastatin is one of the FDA approved statin drugs. High first pass effect also causes dominant effect of Simvastatin in the liver. Owing to the adverse effects of statins, considerable research interest has been shifted towards the hypolipidemic effects of natural products like Phoenix dactylifera (Palm Date). It is being used as diet by humans for almost last 6000 years, hence, one of the oldest plants on earth. Ajwa 
is one type of Palm Dates which are only cultivated in Al-Madinah Al-Munawara, Saudi Arabia. It has special value for Muslims as many Ahadiths are there to highlight its importance. ${ }^{8}$ Ajwa date has been used as diet and remedy for various diseases. Dates are composed of constituents which are of high nutritional value. These include carbohydrate, vitamins, fatty acids, minerals, dietary fibers and various salts.

However, in contrast to the delicious pericarp or date fruit, the date seed is considered usually a waste product. Recently, it is mostly used as a feed for animals like camel, sheep, poultry and cattle. ${ }^{9}$ Limited research has been done on the benefits of the date seeds.

According to a study, Ajwa date seed was found to contain almost $19 \%$ crude fiber, $7.8 \%$ crude proteins and $9.8 \%$ crude fat. It also contained $62 \%$ nitrogen Free Extract (NFE) which denoted carbohydrates, starches and sugars. Ajwa date seed was also enriched with various minerals like phosphorous, potassium, zinc, boron, iron, magnesium, copper, calcium and sodium. HPLC profile of Ajwa date seed also showed promising antioxidant content containing high amount of phenolic compounds and flavonoids. It included presence of gallic acid, caffiec acid, syringic acid, coumeric acid, ferulic acid, chromatotrophic acid, quercetin and vitamin C. ${ }^{10}$ Being a rich source of antioxidants, Ajwa date seed is believed to possess vital role in the prevention and treatment of various diseases. It showed hepatoprotective effects in rat model of hepatotoxicity due to CCL4 in which levels of ALT and AST (markers of liver functions) significantly decreased. ${ }^{11}$ Most of these effects are attributed to the antioxidant potential of Ajwa date seeds. For investigation of hepatoprotective effects of Ajwa date seed and Simvastatin against fatty liver disease, current research was undertaken.

\section{MATERIAL AND METHODS}

A 36 day Study was conducted in Experimental Research Laboratory of UHS, Lahore. Biochemical tests for lipid profile were performed at Biochemistry Lab of Shaikh Zayed Hospital, Lahore.

Animals: 40 healthy male albino rats weighing in the range of $100-150 \mathrm{gms}$ were purchased from University of Veterinary and Animal Sciences, Lahore. They were segregated through lottery method into 5 groups of 8 animals in each group. The animals were kept in the animal house at UHS, Lahore and were given free access to standard laboratory diet.
Animal diet: Standard animal diet was procured from the University of Veterniary and Animal Sciences (UVAS) Lahore. For preparation of high fat diet (HFD), Adam's butter 250grams was added to standard laboratory diet to make 1000grams of high fat diet (HFD).

Plant material: Ajwa dates were purchased from the Irum Mart, Walton Road, Lahore imported from Madinah. Date seeds of above mentioned mart were used throughout the experiment.

Aqueous Ajwa Date Seed Extract (ADSE) preparation: Fruit pulp was removed and seed were separated, washed and dried at room temperature for 2-3 days. Dry seeds were roasted in the Home Coffee Roasting Machine for $15 \mathrm{~min}$ at $220^{\circ} \mathrm{C}$ and then cooled to room temperature. ${ }^{10}$ Roasted seeds were crushed into small pieces and powder was made after grinding in the coffee grinder. 100 grams of seed powder was soaked in 1 liter of distilled water and kept at $4^{\circ} \mathrm{C}$ for 48 hours with intermittent stirring. The extract was filtered through cotton after 48 hours and the supernatant was further used in the research project. Dose of aqueous Ajwa Date Seed Extract was administered in amount of $8 \mathrm{ml} / \mathrm{kg} /$ day. ${ }^{12}$ According to the reference article, this dose of ADSE produced the most favorable effects without any evident adverse effects in rats.

Chemicals and Drugs: Simvastatin 10mg tablet was purchased from Clinix pharmacy Jail Road, Lahore. It was administered orally suspended in distilled water in the dose of $10 \mathrm{mg} / \mathrm{kg} / \mathrm{day}$.

Grouping of Animals: Animals were segregated into 5 groups with 8 rats in each group.

Group 1 (Healthy Control group) animals were given the normal laboratory diet for the entire study period of 36 days, and left untreated.

Group 2 (Disease Control group) was fed butter for 36 days and left untreated as well.

All other groups (3-5) were butter fed for entire study period of 36 days and administered drugs through gastric gavage for 21 days after the induction of dyslipidemia at day 15.

Group 3: Simvastatin 10mg/ kg/day

Group 4: Ajwa Date Seed extract $8 \mathrm{ml} / \mathrm{kg} /$ day

Group 5: Simvastatin 5mg/kg/day and Ajwa Date Seed extract $4 \mathrm{ml} / \mathrm{kg} / \mathrm{day}$

Biochemical tests: Blood sampling was done at day 0,15 and 36 through intracardiac puncture. Serum was collected by centrifugation and biochemical tests (serum TC, TG, ALT, AST and ALP) were performed by using biochemical kits of Siemens Company. 


\section{Statistical analysis:}

Data was analyzed using SPSS version 23.0. Comparison among groups was made by use of one way ANOVA and posthoc Tuckey's test.

\section{RESULTS}

At the start of study, all biochemial parameters (serum TC, TG, ALT, AST and ALP) were in the normal range.

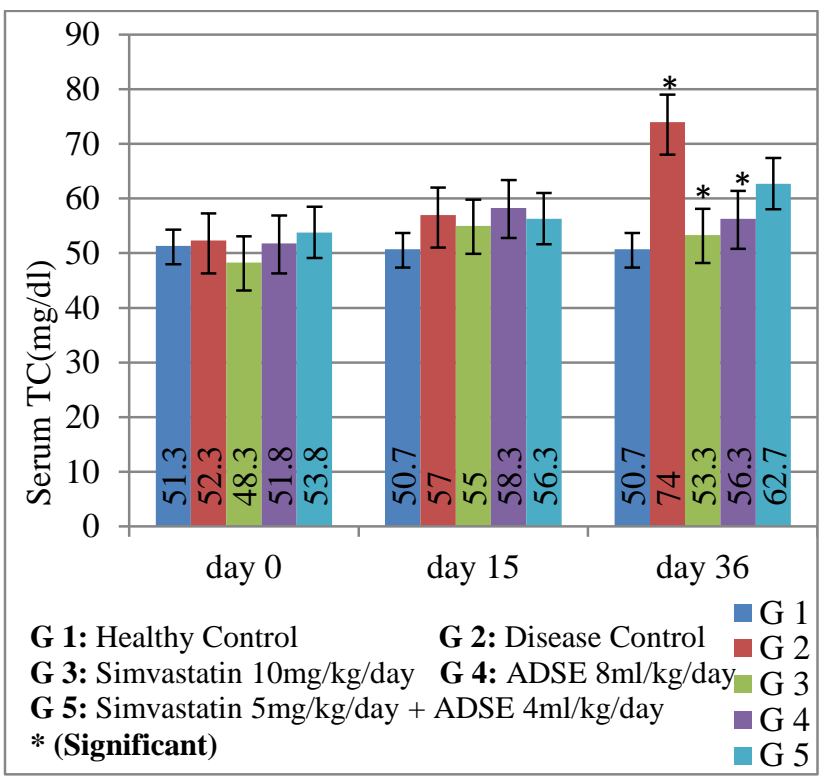

Fig-1: Serum TC of groups 1, 2, 3, 4 and 5 on day 0,15 and $36(\mathrm{n}=8)$

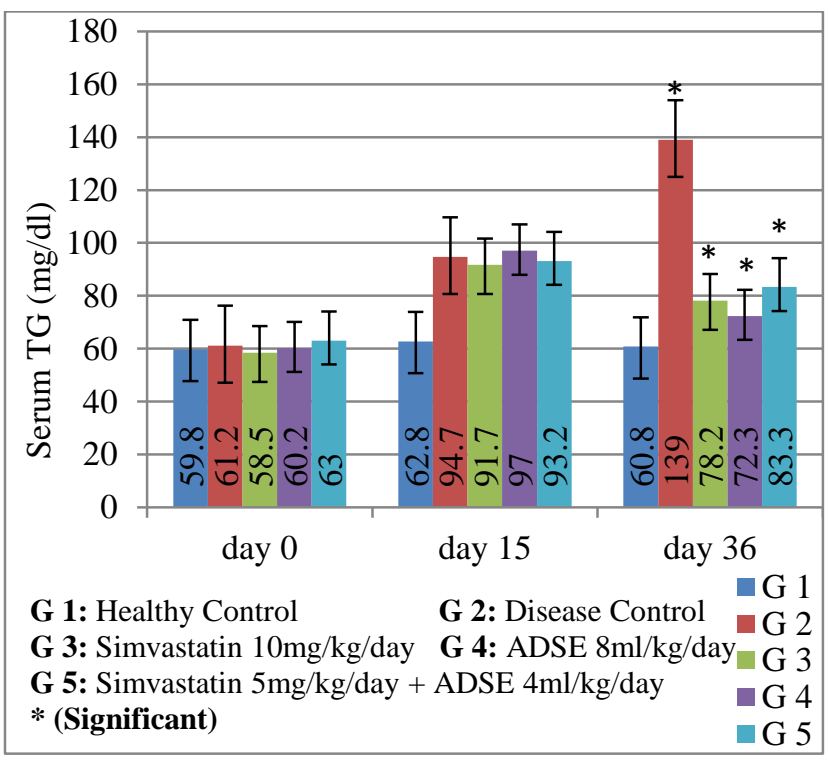

Fig-2: Serum TG $(\mathrm{mg} / \mathrm{dl})$ of groups $1,2,3,4$ and 5 on day 0,15 and $36(n=8)$

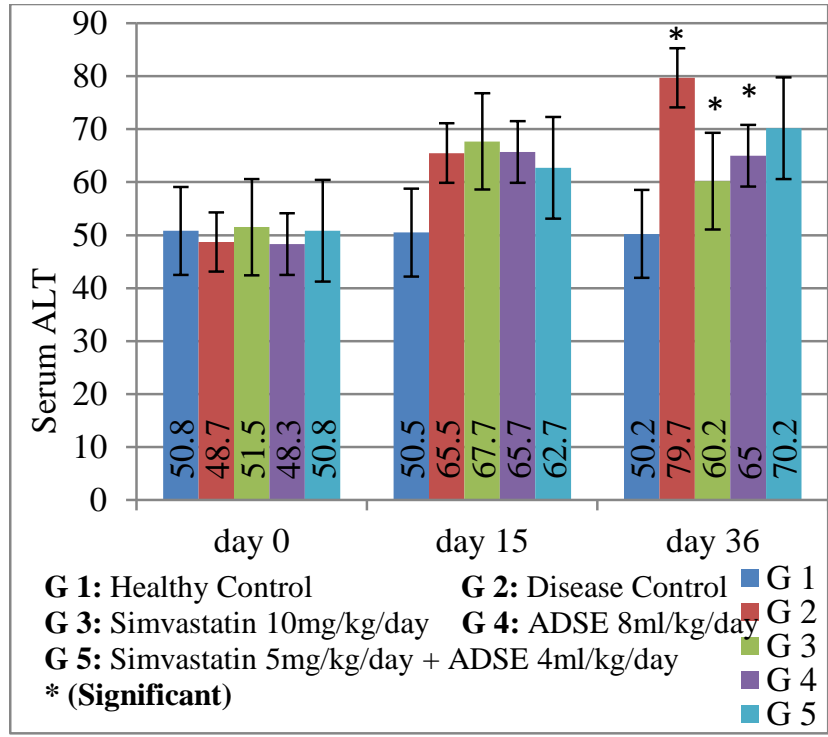

Fig-3: Serum ALT (mg/dl) of groups 1, 2, 3, 4 and 5 on day 0,15 and $36 \quad(n=8)$

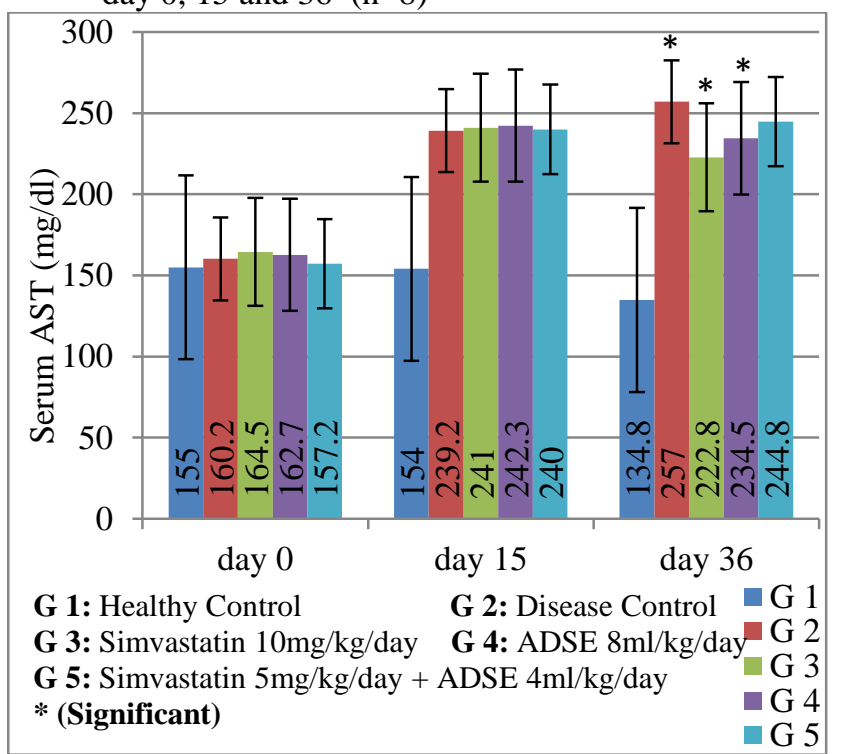

Fig-4: Serum AST (mg/dl) of groups 1, 2, 3, 4 and 5 on day 0,15 and $36 \quad(n=8)$

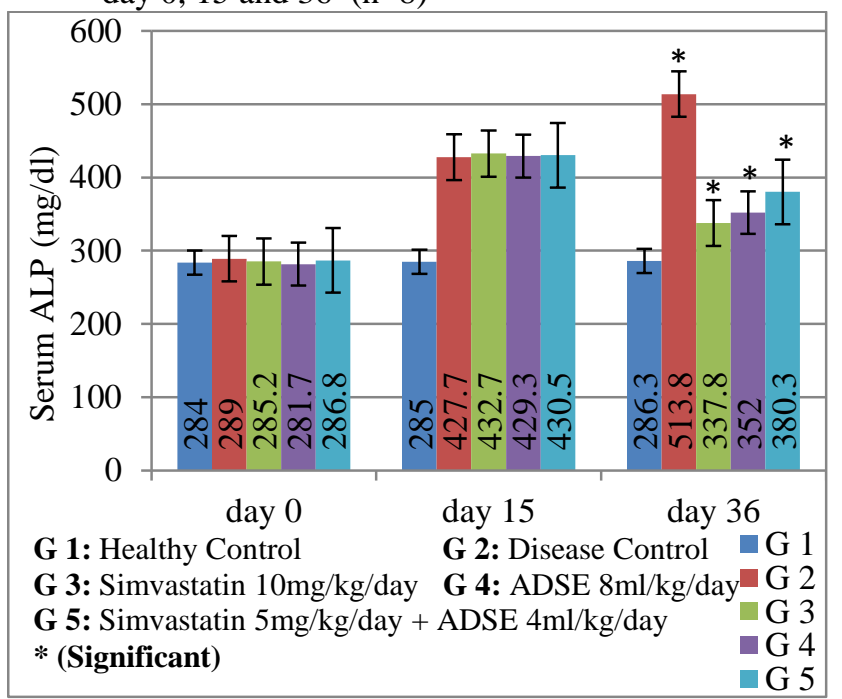

Fig-5: Serum ALP (mg/dl) of groups 1, 2, 3, 4 and 5 on day 0,15 and $36(n=8)$ 
After butter feeding for 15 days, there was significant increase in lipid parameters (TC and TG) and LFTs (ALT, AST and ALP). Treatment with Simvastatin $10 \mathrm{mg} / \mathrm{kg} /$ day and aqueous ADSE $8 \mathrm{ml} / \mathrm{kg} /$ day significantly brought the deranged lipid profile parameters and LFTs towards normalization as compared to disease control group. Combination of drugs (simvastatin $5 \mathrm{mg} / \mathrm{kg} / \mathrm{day}+\mathrm{ADSE}$ $4 \mathrm{ml} / \mathrm{kg} /$ day) also improved all parameters but significant effects were found only on serum TG and ALP when compared with disease control group. P-value was less than 0.05 which was considered significant. Results are displayed in the above figures 1-5.

\section{DISCUSSION}

In current research work, impact of aqueous Ajwa date seed extract (ADSE) 8ml/kg/day, Simvastatin $10 \mathrm{mg} / \mathrm{kg} /$ day and their combination in half doses on non-alcoholic fatty liver disease (NAFLD) due to butter feeding was assessed on total cholesterol, triglycerides and on biochemical parameters of liver injury known as liver function tests (ALT, AST and ALP).

Upon conclusion of experiment, disease control group had significant derangement of serum TC and TG as it was given no treatment. Continuous butter feeding for 36 days caused excessive lipid absorption from intestines and elevated levels of lipids in the blood. ${ }^{4}$ Dyslipidemia is a known cause of fatty liver disease which led to accumulation of cholesterol in the cytoplasm of hepatocytes as fat droplets (steatosis). Fat accumulation inside the hepatocytes resulted in their ballooning and disrupted the cellular structure of hepatocytes. ${ }^{13}$ Injury to hepatocytes was manifested by increased levels of liver function tests (ALT, AST and ALP). In our study, administration of butter containing diet significantly deranged the LFTs of rats. It was also ratified in another study where high fat diet consumption also caused liver injury and deranged LFTs. $^{4}$

Treatment with Simvastatin $10 \mathrm{mg} / \mathrm{kg} /$ day reduced the serum TC and $\mathrm{TG}$ by $3 \%$ and $14 \%$. It also normalized the serum ALT, AST and ALP significantly by $12.5 \%, 8 \%$ and $29 \%$. Effects of Simvastatin were significant when compared to disease control group as p-value was less than 0.05. Simvastatin is a known antihyperlipidemic drug. It interferes with hepatic cholesterol production by inhibiting the HMG CoA reductase enzyme which catalyzes the rate limiting step of cholesterol synthesis. In response, LDL receptors on the surface of hepatocytes are up regulated by genetic transcription to maintain hepatic cholesterol levels. It results in low lipid levels in blood. ${ }^{14}$ Subsequently, statin mediated inhibition of intrahepatic cholesterol synthesis cannot be compensated by extrahepatic sources and hepatic cholesterols stores decline. Reduced cholesterol levels results in resolution of steatosis and normalize the cellular structure of hepatocytes, thus, decreasing release of markers of hepatocyte injury exhibited as declined LFTs. ${ }^{15}$ Effects of Simvastatin $10 \mathrm{mg} / \mathrm{kg} /$ day on hyperlipidemia and non-alcoholic fatty liver disease were validated in current study.

Interestingly aqueous ADSE $8 \mathrm{ml} / \mathrm{kg} /$ day showed comparable results to standard drug Simvastatin. At the end of study, it decreased the serum TC and TG levels by $4 \%$ and $34 \%$. Effects of aqueous ADSE on normalization of serum ALT, AST and ALP were also remarkable. P-value was less than 0.05 which shows significant association of ADSE with normalization of lipid profile parameters and LFTs as compared to disease control group. The reason could be that Ajwa date seed contains high amount of antioxidant compounds polyphenols (Gallic acid, Chlorogenic acid, Ferulic acid, Coumaric acid, Caffiec acid, Catechin and Querctin), and vitamin C which have been found in citrus peel extract as well. ${ }^{10}$ In a previous research, these antioxidant compounds reduced the activity of hepatic enzymes 3-hydroxy-3-methyl-glutaryl-CoA (HMG CoA) reductase which is vital for cholesterol biosynthesis. ${ }^{16,17}$ Antioxidants inhibit the HMG CoA reductase enzyme through non-competitive inhibition by modification of binding site of enzyme resulting in blockage of enzyme-substrate complex. ${ }^{18}$ Antioxidants especially polyphenols and vitamin $\mathrm{C}$ contained in Ajwa date seed may be responsible for decrease in hepatic cholesterol stores resulting in increased hepatic uptake of cholesterol from extra hepatic sources especially vascular system. It results in lowering of blood lipid levels. ${ }^{15}$ Quercetin is a phenolic compound present in Ajwa date seed. It blocks the transcription of several enzymes involved in the synthesis of fatty acids. ${ }^{19}$ Saponins are also present in Ajwa date seed which reduces absorption of lipids in intestines and causes inhibition of lipase enzyme. ${ }^{20}$ Resolution of fatty changes in the liver restores the normal hepatic architecture and liver injury markers are normalized. In recent study, Ajwa date seed showed promising results to normalize the serum TC, TG and LFTs of dyslipidemic butter fed rats. This finding is in line with another study in which ADSE reduced the serum TC and TG in diabetic model of rats. ${ }^{20}$ Aqueous ADSE was also found to reduce LFTs in the rats intoxicated with carbon tetrachloride. ${ }^{12}$ 
Combination of drugs (Simvastatin and aqueous ADSE) in half dose also reduced serum TC, TG and LFTs but less than the individual drugs. Moreover, its effects were significant only on serum TG and ALP when compared with disease control group. This may be due to presence of sub therapeutic levels of drugs in the body after reducing the drug amount to half of original dose. No synergistic or additive effects were seen among both drugs.

\section{CONCLUSION}

These biochemical findings suggest total cholesterol, serum triglycerides lowering and hepatoprotective role of Ajwa date seed and Simvastatin against fatty liver disease due to consumption of high fat diet.

\section{REFERENCES}

1. Erickson SK. Nonalcoholic fatty liver disease. Journal of lipid research. 2009; 50 (Supplement) :S412-S6.

2. Beyegue CN, Ngangoum RC, Kuate D, Ngondi J, Oben JE. Effect of Guibourtia tessmannii extracts on blood lipids and oxidative stress markers in triton WR 1339 and high fat diet induced hyperlipidemic rats. Biology and Medicine. 2012; 4(1):1.

3. Temple JL, Cordero P, Li J, Nguyen V, Oben JA. A guide to non-alcoholic fatty liver disease in childhood and adolescence. International journal of molecular sciences. 2016; 17(6):947.

4. El-Sheekh MM, Hamad SM, Gomaa M. Protective effects of Spirulina on the liver function and hyperlipidemia of rats and human. Brazilian Archives of Biology and Technology. 2014; 57(1):77-86.

5. Eslamparast T, Eghtesad S, Hekmatdoost A, Poustchi H. Probiotics and nonalcoholic fatty liver disease. Middle East journal of digestive diseases. 2013; 5(3):129.

6. S Antonopoulos A, Margaritis M, Lee R, Channon K, Antoniades C. Statins as antiinflammatory agents in atherogenesis: molecular mechanisms and lessons from the recent clinical trials. Current pharmaceutical design. 2012; 18(11):1519-30.

7. Lipsy RJ. Overview of pharmacologic therapy for the treatment of dyslipidemia. Journal of Managed Care Pharmacy.2003;9(1SuppA):9-12

8. Shabani F, Kumar L. Sensitivity analysis of CLIMEX parameters in modeling potential distribution of Phoenix dactylifera L. PloS one. 2014; 9(4):e94867.
9. Abdul A, Abdul R, Che M, Al-Kahtani H, Mansor T. Date seed and date seed oil. International Food Research Journal. 2013; 20(5): 2035-43.

10. Ahmed A, Arshad MU, Saeed F, Ahmed RS, Chatha SAS. Nutritional probing and HPLC profiling of roasted date pit powder. Pakistan Journal of Nutrition. 2016; 15(3):229.

11. Sheikh BY, Elsaed WM, Samman AH, Sheikh BY, Ladin A-MMB. Ajwa dates as a protective agent against liver toxicity in rat. European Scientific Journal, ESJ. 2014; 10(7).

12. Al-Rasheed NM, Attia HA, Mohamad RA, AlRasheed NM, Al-Amin MA, AL-Onazi A. Aqueous date flesh or pits extract attenuates liver fibrosis via suppression of hepatic stellate cell activation and reduction of inflammatory cytokines, transforming growth factor- $\beta 1$ and angiogenic markers in carbon tetrachlorideintoxicated rats. Evidence-based complementary and alternative medicine. 2015.

13. Brunt EM, Tiniakos DG. Histopathology of nonalcoholic fatty liver disease. World journal of gastroenterology: WJG. 2010; 16(42):5286.

14. Alizadeh J, Zeki AA, Mirzaei N, Tewary S, Moghadam AR, Glogowska A, et al. Mevalonate Cascade Inhibition by Simvastatin Induces the Intrinsic Apoptosis Pathway via Depletion of Isoprenoids in Tumor Cells. Scientific Reports. 2017; 7:44841.

15. Ahmadi Y, Ghorbanihaghjo A, Argani H. The effect of statins on the organs: similar or contradictory? Journal of cardiovascular and thoracic research. 2017; 9(2):64.

16. Bok S-H, Lee S-H, Park Y-B, Bae K-H, Son K$\mathrm{H}$, Jeong T-S, et al. Plasma and hepatic cholesterol and hepatic activities of 3-hydroxy3-methyl-glutaryl-CoA reductase and acyl CoA: cholesterol transferase are lower in rats fed citrus peel extract or a mixture of citrus bioflavonoids. The Journal of nutrition. 1999; 129(6):1182-5.

\section{The Authors:}

Dr. Farooq Sultan

Demonstrator,

Department of Pharmacology \& Therapeutics, Gujranwala Medical College, Gujranwala.

Dr. Saba Anwar

Women Medical Officer,

Rural Health Centre Hadali, Khushab. 
Prof. Saadia Shahzad Alam

HOD Pharmacology \& Therapeutics,

Shaikh Zayed Medical Complex, Lahore.

Dr. Hassan Farooq

Assistant Professor,

Department of Pharmacology \& Therapeutics,

Khawaja Muhammad Safdar Medical College, Sialkot.

Prof. Samina Kausar

Department of Pharmacology \& Therapeutics, Gujranwala Medical College, Gujranwala.
Dr. Tehreem Zulfiqar

Assistant Professor,

Department of Pharmacology \& Therapeutics, Nishtar Medical University Multan.

\section{Corresponding Author:}

Dr. Farooq Sultan

Demonstrator,

Department of Pharmacology \& Therapeutics, Gujranwala Medical College, Gujranwala.

E-mail: farooqsultan2000@gmail.com 